\title{
Liderazgo y satisfacción laboral en la Facultad de Ciencias Contables, Financieras y Administrativas, Universidad Católica los Ángeles de Chimbote
}

\section{Leadership and job satisfaction at the Facultad de Ciencias Contables, Financieras y Administrativas, Universidad Católica los Ángeles de Chimbote}

\author{
María Isabel Mino Asencio* \\ Víctor Julio Zavaleta León ${ }^{* *}$
}

\section{RESUMEN}

\begin{abstract}
$A^{1}$ l ejercer liderazgo, las personas adoptan "estilos" que probablemente puedan tener cierta influencia en la satisfacción laboral de los trabajadores, lo que puede incidir en el trabajo que desempeñan, por lo que esta investigación tuvo como objetivo determinar la relación entre los estilos de liderazgo y la satisfacción laboral del personal administrativo y docente de la Facultad de Ciencias Contables, Financieras y Administrativas de la Universidad Católica Los Ángeles de Chimbote. Se aplicó, a una muestra de 30, entre docentes, administrativos y directivos de la facultad, una encuesta que consta de tres partes: datos generales, evaluación de los estilos de liderazgo y evaluación de la satisfacción laboral del personal de la facultad.

Se concluyó que los estilos de liderazgo predominantes son el transaccional y transformacional; la satisfacción laboral global del personal docente y administrativo es alta, sin dejar de tener en consideración que dentro de los factores extrínsecos, los ítems referentes al horario de trabajo y remuneración han mostrado una satisfacción baja, y que existe una correlación alta entre el liderazgo y la satisfacción laboral del personal docente y administrativo de la Facultad de Ciencias Contables, Financieras y Administrativas de la Universidad Católica Los Ángeles de Chimbote.
\end{abstract}

Palabras claves: liderazgo, satisfacción laboral.

* Facultad de Ciencias Contables, Financieras y Administrativas. Uladech Católica, Chimbote. isabel_mino_asencio@hotmail.com

** Facultad de Ciencias Económicas - UNT. aymerick_10@hotmail.com 


\begin{abstract}
While exercising leadership, people adopt "styles" that probably may have some influence on the job satisfaction of workers, which can have an impact on the work, so the goal of this research was to determine the relation between leadership styles and the satisfaction of administrative staff and teachers of the Faculty of Accounting, Financial and Administrative Sciences of the Universidad Católica Los Ángeles of Chimbote. A survey was applied to a sample of 30, between administrative personnel, managers and teachers of the faculty. That survey had three parts: general data, assessment of leadership styles and job satisfaction of the faculty staff.

In conclusion, the main leadership styles are the transactional and transformational; overall job satisfaction of the teaching and administrative staff is high, while taking into consideration that, regarding outer factors, the items related to the hours of work and salary have shown a low satisfaction, and there is a high correlation between leadership and job satisfaction of teachers and administrative personnel of the Faculty of Accounting, Financial and Administrative Sciences of Universidad Católica Los Ángeles de Chimbote.
\end{abstract}

Keywords: leadership, job satisfaction. 


\section{INTRODUCCIÓN}

De acuerdo con Ahumada (2004), el contexto en el que se insertan las organizaciones durante las últimas décadas se ha caracterizado por cambios discontinuos e impredecibles, y la consiguiente incertidumbre organizacional. Esta evidente e incesante adaptación de las empresas a su ambiente y el innegable hecho de que deban competir con otras es un aspecto que hace que el tema del liderazgo preocupe cada vez más a la teoría y práctica organizacional, dado que del buen ejercicio de este se obtendrían mejores indicadores de eficacia y competitividad, además de constituirse en "uno de los elementos más representativos de la dinámica que caracteriza el ser y el quehacer organizacional actual".

En la tesis de Vega V., Carolina; Gloria Zavala (2004) se define "liderazgo" como "el proceso de influencia que un individuo ejerce sobre otro individuo o grupo, para lograr el alcance de objetivos en una determinada situación". En este mismo trabajo se cita una investigación llevada a cabo en 1948 por Stogdill, ampliada y revisada 25 años más tarde, donde se demuestra que no existe un grupo de características que definan universalmente el liderazgo. En 1974, el mismo autor concluyó que las características y habilidades que requiere el liderazgo vienen determinadas por una situación específica. Consciente o inconscientemente, el liderazgo se lleva a la práctica de maneras diferentes, dependiendo de la situación, de la disposición de los poderdantes y según las características propias del mismo líder, adoptando "estilos de liderazgo".

El ejercicio del liderazgo, a pesar de ser complejo, es inherente a la organización del trabajo y repercute en la articulación de los equipos. Al efectuar su función, un líder debe acercarse a un estilo en particular y entre estos destacan, según el modelo de Hersey y Blanchard (1998), el director, entrenador, participativo / apoyador y delegador. Con esto se debe entender que el modelo o estilo adoptado define la eficacia del líder en función de su capacidad para utilizar cada uno de los estilos de acuerdo con la situación de los colaboradores. No existe un estilo de liderazgo que sea siempre correcto de utilizar en todas las situaciones, por tanto, el líder más eficaz será aquel que demuestre una mayor flexibilidad de estilo y que utilice el estilo adecuado con la persona adecuada. Según Hersey et al. (1998), a cada nivel de preparación le correspondería un estilo de liderazgo en concreto.

Ahora bien, según Galaz (2002), liderazgo se entiende como la "capacidad de influir en un grupo para que consiga sus metas", pero independientemente de su conceptualización, el tema del liderazgo es uno de los que más investigación ha generado en el ámbito de la psicología social de las organizaciones, debido a su consideración como factor significativo en el funcionamiento y éxito de las empresas.

Además, de la variable "satisfacción laboral" podemos decir que es una actitud y que, si bien se han propuesto diferentes definiciones, buena parte de ellas han puesto el énfasis 
en el componente afectivo de las actitudes (las emociones o sentimientos que suscita el objeto actitudinal), sin considerar como se debería, los elementos cognitivos (creencias sobre las características positivas o negativas del objeto actitudinal) y conductuales (conductas o experiencias pasadas relacionadas con el objeto de actitud). Alargunsoro (2012)

A decir de Galaz (2002), al ejercer liderazgo, las personas adoptan "estilos" que probablemente puedan tener cierta influencia en la satisfacción laboral de los trabajadores, lo que puede incidir en el trabajo que desempeñan.

El grado de satisfacción puede afectar la cantidad y calidad del trabajo que desempeñan los sujetos, e incidir en la baja calidad de la prestación, ausentismo laboral, propensión a abandonar la organización, entre otros (Muchinsky, 2009). La satisfacción laboral es un aspecto que ha sido ampliamente estudiado desde que Hoppock, ya en el año 1935, publicó la primera investigación que hacía un análisis profundo de la satisfacción laboral, determinando que existen múltiples factores que podrían ejercer influencia sobre la satisfacción laboral, como: fatiga, monotonía, condiciones de trabajo y supervisión. La satisfacción laboral se define como el "grado de placer que el empleado obtiene de su trabajo" (Muchinsky, 2009). En la atención de los servicios educativos ha aumentado en importancia, e incluso se podría decir que al determinar si los profesionales se encuentran o no satisfechos, se está midiendo la calidad de la organización y de sus servicios internos (Marriner, 2009). Las intervenciones destinadas a mejorar la práctica diaria del personal de la Facultad de Ciencias Contables, Financieras y Administrativas de la Universidad Católica Los Ángeles de Chimbote debe centrarse en la carga de trabajo, expectativas personales de los trabajadores y satisfacción en el trabajo. Una de las definiciones más usuales y generales de satisfacción en el trabajo, que incluye componentes cognoscitivos y afectivos es la siguiente: "Un estado emocional agradable o positivo que es el resultado de la valoración del trabajo personal o la experiencia que brinda el trabajo" (Gonzales, 2013). Tiene lugar cuando, en virtud de estar involucrado en ciertas actividades dentro de un ambiente de trabajo particular, las necesidades específicas de un individuo son satisfechas.

La Facultad de Ciencias Contables, Financieras y Administrativas de la Universidad Católica Los Ángeles de Chimbote, en su sede central, cuenta con 72 docentes y 19 trabajadores administrativos que dirigen los destinos y la marcha académica e institucional de las carreras de Contabilidad y Finanzas, Administración Turística, y Administración.

La presente investigación está centrada en determinar los estilos de liderazgo que prevalecen en la organización de la Facultad de Ciencias Contables, Financieras y Administrativas de la Universidad Católica Los Ángeles de Chimbote y relacionar esta variable con la satisfacción laboral que puedan experimentar los trabajadores tanto docentes como administrativos de esta facultad.

Y a nivel empresarial es de vital importancia que estos gocen, entre otras cosas, de buena satisfacción laboral, ya que su carencia contribuye al aumento de factores adversos que implican consecuencias costosas para la empresa, los cuales se podrían ver reducidos si los líderes de la organización favorecen la satisfacción en el trabajo a través de una adecuada organización y gestión. Por tanto, influirá en la satisfacción laboral el estilo de liderazgo percibido por los trabajadores de la Facultad de Ciencias Contables, Financieras 
y Administrativas de la Universidad Católica los Ángeles de Chimbote, probablemente, en la medida en que cada estilo sea ejercido o no, según la situación, el nivel de satisfacción laboral se verá afectado, premisa bajo la cual se instala el propósito central de esta investigación.

De esta forma, al relacionar el estilo utilizado por los líderes de la facultad y la satisfacción de los seguidores respecto a su trabajo, probablemente se pondrán de manifiesto una serie de antecedentes o estrategias que permitan la creación de un buen clima laboral, en el cual prime la comunicación y coordinación entre puestos jerárquicos y el personal docente y administrativo, el cumplimento de objetivos, metas y resultados esperados al tener equipos satisfechos, la identificación de metodologías y planes que fomenten la confianza del grupo, el desarrollo de las capacidades del personal de trabajo, la valoración del profesional como miembro importante del equipo multidisciplinar, así como también su participación activa en todas las áreas de desempeño profesional. Mantener niveles altos de satisfacción laboral permite mejorar los procesos, fomenta el trabajo en equipo, aumenta la calidad de la atención a los estudiantes y el rendimiento de su productividad, así como la satisfacción de los usuarios internos y externos.

Por lo que nos planteamos el siguiente problema de investigación:

¿Cuál es la relación entre los estilos de liderazgo y la satisfacción laboral del personal administrativo y docente de la Facultad de Ciencias Contables, Financieras y Administrativas de la Universidad Católica Los Ángeles de Chimbote - Chimbote, 2014 ?

\section{MATERIAL Y MÉTODO}

Población de estudio: Estuvo conformada por el personal docente y administrativo de la Facultad de Ciencias Contables, Financieras y Administrativas, y se constituyó de la siguiente manera:

\begin{tabular}{lllc}
\hline & \multicolumn{3}{c}{ CONGLOMERADOS } \\
\cline { 3 - 4 } E & \multicolumn{1}{c}{ Administración } & Contabilidad \\
\cline { 3 - 4 } T & Decano & 1 & 1 \\
\cline { 2 - 4 } R & Director & 28 & 40 \\
A & Docentes & 7 & 8 \\
T & Administrativos & 36 & 49 \\
S & Total & &
\end{tabular}

Total $=95$ 


\section{Muestra}

Se aplicó un muestreo estratificado simple y al azar, utilizando la fórmula para poblaciones finitas y conocidas:

Donde:

$\mathrm{N}$ : tamaño de población $=91$

n: tamaño de muestra

e: error de muestra $=0,05$

Z: coeficiente de confianza $=1,96$

p: evento favorable $=0,5$

q: evento no favorable $=0,5$

Luego:

$$
\mathrm{n}=30
$$

La muestra por estratos y conglomerados queda constituida de la siguiente manera:

Muestreo según estrato docente y por conglomerado Muestreo según estrato administrativo y por conglomerado

\begin{tabular}{lccc}
\hline DOCENTES & $\mathrm{N}$ & $\%$ & $\mathbf{n}_{2}$ \\
\hline Administración & 28 & 38,89 & $\mathbf{9}$ \\
Contabilidad & 40 & 55,56 & $\mathbf{1 3}$ \\
Administración Turística & $\mathbf{4}$ & 5,56 & $\mathbf{1}$ \\
\hline TOTAL MUESTRA & 72 & 100 & $\mathbf{2 4}$ \\
\hline ADMINSTRATIVOS & $\mathrm{N}$ & $\%$ & $\mathbf{n}_{2}$ \\
\hline Administración & 7 & 36,84 & $\mathbf{2}$ \\
Contabilidad & 8 & 42,11 & $\mathbf{3}$ \\
Administración Turística & 4 & 21,05 & $\mathbf{1}$ \\
\hline TOTAL MUESTRA & 19 & 100 & $\mathbf{6}$ \\
\hline
\end{tabular}

\section{MÉTODOS, TÉCNICAS Y PROCEDIMIENTOS}

Métodos: Bernal (2006: 57)

- Método deductivo: se utilizó el método analítico-deductivo, ya que se ha trabajado con conclusiones generales de investigaciones previas acerca de liderazgo y de satisfacción laboral en otros contextos para fundamentar nuestro trabajo y poder llegar a particularidades para correlacionar las dos variables de estudio.

- Método inductivo: hemos utilizado este método al momento de describir en específico cada variable de estudio inserta en el trabajo de investigación. (Hernández Sampieri, 2010). 


\section{Técnicas e instrumentos de recolección de datos}

Se utilizaron las siguientes técnicas de recolección de información:

- Encuestas: esta encuesta se fundamenta en un cuestionario o conjunto de preguntas que se han preparado con la intención de recoger información sobre los estilos de liderazgo y la satisfacción laboral del personal docente y administrativo de la Facultad de Ciencias Contables, Financieras y Administrativas de la ULADECH - Católica. Consta de tres partes:

Parte I: Identificación del personal docente y administrativo respecto a su centro laboral y su condición de trabajo.

Parte II: Evaluación de los estilos de liderazgo: se ha preparado un cuestionario de liderazgo multifactorial (MLQ-5S) corto que consta de 45 preguntas con cinco posibles respuestas, según como sigue:

Este cuestionario permite identificar tres tipos de liderazgo: transformacional, transaccional y laissez-faire. De los 45 ítems que comprende, 20 corresponden a liderazgo transformacional, 12 corresponden a liderazgo transaccional y 13 a liderazgo laissez-faire, según como sigue

A. Liderazgo transformacional: se evalúa de la sumatoria del puntaje directo obtenido en los ítems de las escalas: influencia idealizada: está conformada por 8 ítems: 6 , 10, 14, 18, 21 , 23, 25 y 34 del MLQ; motivación inspiracional: está conformada por 4 ítems: 9, 13, 26 y 36 del MLQ; estimulación intelectual: está conformada por 4 ítems: 2, 8, 30 y 32 del MLQ; influencia idealizada atribuida: está conformada por 4 ítems 15, 19, 29 y 31 del MLQ.

A. Liderazgo transaccional: ee obtiene de la sumatoria del puntaje directo obtenido en las escalas: consideración individualizada: está conformada por 9 ítems: 1, 11, 35, 37, 38, 41, 42, 44 y 45 del MLQ; recompensa contingente: está conformada por 3 ítems: 16, 39 y 43 del MLQ.

B. Liderazgo correctivo / evitador: se obtiene de la sumatoria del puntaje directo obtenido en las escalas: dirección por excepción pasiva: está conformada por 9 ítems: 3 , 4, 12, 20, 22 , 24, 27, 33 y 40 del MLQ; liderazgo pasivo / evitador o laissez-faire: está conformada por 4 ítems: 5, 7, 17 y 28 del MLQ.

Parte III: Evaluación de la satisfacción laboral: Se ha preparado un cuestionario de 12 ítems siguiendo la Escala General de Satisfacción (Overall Job Satisfaction) (ver anexo). Fue desarrollada por Warr, Cook y Wall en 1979. Las características de esta escala son las siguientes: es una escala que hace operacional el constructo de satisfacción laboral, reflejando la experiencia de los trabajadores de un empleo remunerado. Recoge la respuesta afectiva al contenido del propio trabajo. (Pérez Bilbao, Jesús, 2011): ESCALA DE SATISFACCIÓN (traducción de Overall Job Satisfaction de Warr, Cook y Wall).

Está formada por dos subescalas: subescala de factores intrínsecos: aborda aspectos como el reconocimiento obtenido por el trabajo, responsabilidad, promoción, aspectos relativos al contenido de la tarea, etc. Esta escala está formada por cinco ítems (números 
2, 4, 6, 8 y 10). Y subescala de factores extrínsecos: indaga sobre la satisfacción del trabajador con aspectos relativos a la organización del trabajo, como el horario, la remuneración, las condiciones físicas del trabajo, etc. Esta escala la constituyen siete ítems (números 1, 3, 5, 7, 9, 11 y 12).

A cada ítem, los encuestados respondieron en una escala de Likert de 5 puntos que va desde "muy insatisfecho" hasta "muy satisfecho".

Se analizó de acuerdo con los siguientes rangos: satisfacción baja: 1,00 - 2,33; satisfacción media: 2,34 - 3,66; satisfacción alta: 3,67 - 5,00.

Se obtuvo un alto índice de confiabilidad del instrumento, a través del Coeficiente de Correlación Alfa de Cronbach. Este fue de 0,927.

\section{RESULTADOS}

$$
\begin{aligned}
& \text { A }=\text { Liderazgo transformacional } \\
& B=\text { Liderazgo transaccional } \\
& C=\text { Liderazgo laissez-faire }
\end{aligned}
$$

Fuente: encuestas aplicadas a personal docente y administrativo de ULADECH-Chimbote, 2014

Tabla 1. Niveles de satisfacción laboral global del personal docente y administrativo de la facultad de ciencias contables, financieras y administrativas de la uladech católica de chimbote, 2014

\begin{tabular}{lcc}
\hline SATISFACCIÓN LABORAL & N. $^{\circ}$ & $\%$ \\
\hline GLOBAL & 1 & 3,33 \\
Baja & 12 & 40,00 \\
Alta & 17 & 56,67 \\
\hline TOTAL & 30 & 100,00 \\
\hline
\end{tabular}

Fuente: encuestas aplicadas a personal docente y administrativo ULADECH-Chimbote. Agosto, 2014 
Tabla 2. Niveles de satisfacción laboral intrínseca

\begin{tabular}{lcc}
\hline SATISFACCIÓN INTRÍNSECA & $\mathbf{N} .^{\circ}$ & $\mathbf{\%}$ \\
Baja & 2,00 & 6,67 \\
Media & 13,00 & 43,33 \\
Alta & 15,00 & 50,00 \\
\hline TOTAL & $\mathbf{3 0 , 0 0}$ & $\mathbf{1 0 0}$ \\
\hline
\end{tabular}

Fuente: encuestas aplicadas a personal docente y administrativo ULADECH-Chimbote, 2014

Tabla 3. Niveles de satisfacción laboral extrínseca

\begin{tabular}{|c|c|c|}
\hline SATISFACCIÓN EXTRÍNSECA & $\mathbf{N} .^{\circ}$ & $\%$ \\
\hline Baja & 1 & 3,33 \\
\hline Media & 12 & 40,00 \\
\hline Alta & 17 & 56,67 \\
\hline TOTAL & 30 & 100 \\
\hline
\end{tabular}

Fuente: encuestas aplicadas a personal docente y administrativo de Uladech-Chimbote, 2014

Tabla 4. Relación entre satisfacción laboral y tipo de liderazgo en la Facultad de Ciencias Contables, Financieras y Administrativas de la Uladech Católica de Chimbote, 2014

\begin{tabular}{lcc}
\hline \multirow{2}{*}{ SATISFACCIÓN } & TIPOS DE LIDERAZGO & \\
\cline { 2 - 3 } & $\mathrm{A}$ & $\mathrm{B}$ \\
\hline Baja & 2 & 4 \\
Media & 12 & 8 \\
Aalta & 16 & 18 \\
\hline Total & 30 & 30 \\
\hline
\end{tabular}

Fuente: encuestas aplicadas a personal docente y administrativo Uladech, Chimbote. Agosto, 2014 


\section{DISCUSIÓN}

De los resultados obtenidos encontramos la distribución del personal docente y administrativo de la ULADECH Católica en Chimbote, donde podemos evidenciar que el $80 \%$ son docentes y $20 \%$ administrativos, esto se debe a la naturaleza de la institución, que es de educación, y los más de sus trabajadores son docentes por el servicio educativo que se brinda. De estos docentes, el 73,3\% son hombres y la diferencia son mujeres, por lo que se ve un marcado predominio del sexo masculino debido a la naturaleza de las carreras estudiadas que comúnmente albergan mayor número de profesionales varones. Las edades oscilan entre 31 y 40 años, lo que nos demuestra que es una muestra bastante joven, lo que garantiza el dinamismo y la predisposición al cambio propios de la juventud; sin embargo, también por la juventud son más exigentes y sus parámetros de calidad y satisfacción aumentan, al igual que las expectativas que tienen, ya que son jóvenes y se están consolidando familiar y profesionalmente. En cuanto a la modalidad de trabajo, la mayoría labora a tiempo parcial y por contrato, lo que no garantiza su permanencia, ni estabilidad, y esto puede ser contraproducente para la institución, ya que no permite el fomento de la identidad ni el nexo contractual con ella.

Se obtuvo un alto índice de confiabilidad del instrumento a través del Coeficiente de Correlación Alfa de Cronbach. Este fue de 0,927, lo que indica que en la prueba, los ítems pertenecen al mismo dominio muestral y se están controlando las fuentes de error debidas al muestreo aleatorio.

Se muestra cómo es que el personal docente y administrativo de la Facultad de Ciencias Contables, Financieras y Administrativas de la ULADECH percibe los estilos de liderazgo existentes en la institución, y se encontró que el liderazgo que predomina es el transaccional con una percepción alta en la escala de Likert del 60 \% de encuestados, seguido por el liderazgo tranformacional con un 50 \% de percepción alta, frente al liderazgo laissez-faire con una percepción en su mayoría media y baja.

Describiremos las dos características del liderazgo transaccional: la recompensa contingente y la dirección por excepción. En cuanto a la recompensa contingente, el líder y seguidor acuerdan qué debe hacerse para alcanzar la recompensa o evitar la sanción. Los seguidores continúan si sienten el intercambio transparente y las distribuciones otorgadas equitativamente (Vega \& Zavala, 2004: 106).

La recompensa contingente aprecia cómo las relaciones de negociación son importantes, pero basadas en el castigo y premios, los cuales son materiales o inmateriales para asegurar la participación activa del seguidor en las acciones de líder transaccional.

En términos de Bass y Avolio, la conducta del líder refuerza a los seguidores en el cumplimiento de sus obligaciones y objetivos. La recompensa es próvida en intercambio de las demandas del líder. La segunda característica es la dirección por excepción, definida como la conducta del líder basada en la coerción o castigo de los subordinados identificando sus errores. Dicha posición se complementa cuando el líder toma decisiones sobre las fallas y desviaciones del desempeño esperado (Vega \& Zavala, 2004: 117). 
La dirección por excepción corrige sobre la base de los defectos de los colaboradores. La corrección no fomenta una mayor interrelación entre seguidores y líderes, por el contrario, disminuyen la percepción del desempeño (Bass, 1985) del seguidor y su utilidad para la institución.

En la ULADECH Católica se reconoce este tipo de liderazgo, ya que el personal se ve agobiado por la presión del cumplimiento de metas de la institución, como por ejemplo, el "ranking docente", que califica a los docentes en relación con su desempeño y les da un orden de ubicación y en función de ello se les otorga una serie de beneficios como capacitación, aumento en la carga horaria y, con ello, el aumento de salario; y el no cumplimiento de las metas los hace merecedores de castigos como reducción de su carga horaria, devolución de los costos de las capacitaciones otorgadas, etc.

En cuanto al líder transformacional, es el único capaz de operar a todos los niveles y desplegar todas las conductas de liderazgo, pues su nivel de desarrollo le permite entender la perspectiva de las otras formas de liderazgo.

Bass define al liderazgo transformacional como un proceso que se da en la relación líder-seguidor, en la que el líder se caracteriza por ser carismático, de tal forma que los seguidores se identifican y desean emularlo. Es intelectualmente estimulante, expande las habilidades de los seguidores; los inspira, a través de desafíos y persuasión, proveyéndoles significado y entendimiento. Finalmente, considera a los subordinados individualmente, proporcionándoles apoyo, guía y entrenamiento (Bass y Avolio, 1994).

Los líderes poseedores de inteligencia emocional son apreciados por sus coetáneos debido a su habilidad para asumir el lugar de los demás, de modo tal que puedan anticiparse a los actos de los otros. Es decir, son personas capaces de refrenar sus impulsos emocionales, interpretar los sentimientos más íntimos de los demás y mantener relaciones de una manera fluida.

Es importante señalar que, como lo propone el modelo de Hersey y Blanchard (1998), no existe un estilo de liderazgo óptimo. Por el contrario, el estilo dependería directamente del nivel de preparación, también denominado nivel de madurez de los seguidores en los que el líder pretende influir, a fin de lograr la consecución de objetivos; por tanto, la satisfacción laboral está muy relacionada con el tipo de supervisión, comunicación, autonomía y liderazgo en las autoridades de la Universidad Católica Los Ángeles de Chimbote.

Las gestores de la Facultad de Ciencias Contables, Financieras y Administrativas de la ULADECH pueden influir ejerciendo un estilo de liderazgo adecuado a la situación y, de esta manera, aumentar la motivación y satisfacción laboral de su personal; como indica el estudio español realizado por Aguilar et al. (2007), el estilo de liderazgo que se adopte puede influir en el perfil de valores que tomen los seguidores o subordinados; por tanto, como concluyen en su estudio Rodríguez et al. (2009), resulta primordial prestar atención a los estilos de liderazgo de los diferentes gestores de unidades o de grupos, dado que es un elemento dinamizador importante en las organizaciones educativas.

La tabla 5 muestra los resultados de la evaluación de la consistencia interna del cuestionario usado para medir la satisfacción laboral global basada en el Alfa de Cronbach, 
que nos arroja el dato de 0,895 que es alta confiablidad del cuestionario, como ya se indicó para el cuestionario de liderazgo.

En la tabla 1 podemos evidenciar los niveles de satisfacción laboral global del personal docente y administrativo de la Facultad de Ciencias Contables, Financieras y Administrativas de ULADECH Católica, y encontramos una satisfacción laboral global alta, lo que significa que el personal está satisfecho con el trabajo que desempeña.

La escala utilizada hace operacional el constructo de satisfacción laboral, reflejando la experiencia de los trabajadores de un empleo remunerado, y recoge la respuesta afectiva al contenido del propio trabajo.

La escala está diseñada para abordar tanto los aspectos intrínsecos como los extrínsecos de las condiciones de trabajo. Está formada por dos subescalas: subescala de factores intrínsecos, tabla 2, que aborda aspectos como el reconocimiento obtenido por el trabajo, responsabilidad, promoción y aspectos relativos al contenido de la tarea, y la subescala de factores extrínsecos, tabla 3, que indaga sobre la satisfacción del trabajador con aspectos relativos a la organización del trabajo, como el horario, la remuneración, las condiciones físicas del trabajo, etc.

En cuanto a los factores intrínsecos, los resultados nos muestran un nivel de satisfacción alto (50 \%) y medio (43 \%), lo que significa que el personal siente que está siendo reconocido en su trabajo, y que se puede ver evidenciado a través de la promoción en el empleo, capacitación, asignación de carga horaria extra remunerada, entre otros; y en cuanto a los factores extrínsecos, lo relativo al horario es el punto más álgido encontrado, ya que muestra una satisfacción baja. La remuneración es otro dato con el que no están satisfechos; sin embargo, la satisfacción general de los factores extrínsecos nos permite tener una puntuación alta del $56 \%$.

Estas puntuaciones no suelen incorporar una gran precisión, puesto que su base radica en juicios subjetivos y apreciaciones personales sobre un conjunto más o menos amplio de diferentes aspectos del entorno laboral, y condicionado por las propias características de las personas.

A nivel empresarial, es de vital importancia que el personal goce, entre otras cosas, de buena satisfacción laboral, ya que su carencia contribuye al aumento de factores adversos que implican consecuencias costosas para la universidad, los cuales se podrían ver reducidos si los líderes de la facultad favorecieran la satisfacción en el trabajo a través de una adecuada organización y gestión.

En la Facultad de Ciencias Contables, Financieras y Administrativas de la ULADECH existe una relación altamente significativa entre los tipos de liderazgo transformacional y transaccional y la satisfacción laboral, como lo podemos evidenciar en la tabla 4, es decir, el personal docente y administrativo muestra satisfacción alta para los liderazgos identificados y estas variables están relacionadas directamente.

La correlación entre las dos variables es altamente significativa, según la prueba Rho de Spearman, a un nivel menor de 0,01 bilateral para variables cualitativas. 
De esta forma, al relacionar el estilo utilizado por los líderes de la Facultad de Ciencias Contables, Financieras y Administrativas de la ULADECH y la satisfacción laboral del personal docente y administrativo respecto a su trabajo, se pone de manifiesto una serie de antecedentes o estrategias que permitirían la creación de un buen clima laboral, en el cual prime la comunicación y coordinación entre puestos jerárquicos y personal docente y administrativo, el cumplimento de objetivos, metas y resultados esperados al tener equipos de trabajo satisfechos, la identificación de metodologías y planes que fomenten la confianza del grupo, el desarrollo de las capacidades del personal de trabajo, la valoración del profesional docente y administrativo como miembro importante del equipo multidisciplinar, así como también su participación activa en todas las áreas de desempeño profesional.

Mantener niveles altos de satisfacción laboral permite mejorar los procesos, fomenta el trabajo en equipo, aumenta la calidad de la atención del personal y el rendimiento de su productividad, así como la satisfacción de los usuarios.

\section{CONCLUSIONES}

- Los estilos de liderazgo encontrados en la Facultad de Ciencias Contables, Financieras y Administrativas de la Universidad Católica Los Ángeles de Chimbote con mayor predominio son el liderazgo transaccional y el liderazgo transformacional.

- Se evaluó la satisfacción laboral, y se encontró que el personal docente y administrativo tiene una satisfacción global, sin dejar de tener en consideración que dentro de los factores extrínsecos, los ítems referentes al horario de trabajo y remuneración han mostrado una satisfacción baja.

- Existe una correlación alta entre el liderazgo y la satisfacción laboral del personal docente y administrativo de la Facultad de Ciencias Contables, Financieras y Administrativas de la Universidad Católica Los Ángeles de Chimbote.

\section{REFERENCIAS BIBLIOGRÁFICAS}

Aguilar-Luzón, M., Calvo-Salguero, A., García-Hita M.A. (2007). Valores laborales y percepción del estilo de liderazgo en personal de enfermería. Salud pública México; 49(6): 401-07.

Aguirre, D. (2009). Satisfacción laboral de los recursos humanos de enfermería: factores que la afectan. Disponible en: http://scielo.sld.cu. Consultado el 30 de agosto de 2014. 
Alargunsoro, A.M. (2012). Satisfacción laboral en las enfermeras correturnos de Mutualia durante el año 2011. Biblioteca Las Casas. Disponible en: http:// www. index-f.com/lascasas/documentos/lc0656.pdf. Consultado el 13 de abril de 2014.

Alberoni, Francesco. (1998). Valores. Barcelona: Gedisa. 190 p.

Álvarez de Mon Pan de Soraluce, Santiago y [et al.]. (2001). Paradigmas del liderazgo: claves de la dirección de personas. Madrid: McGraw-Hill Interamericana. 227 p.

Bass, B. (1990). Bass \& Stogdill's handbook of leadership. Theory, research \& managerial applications. USA: The Free Press.

Bass, B. y Avolio, B. (1994). Improving organizational effectiveness through transformational leadership. USA: Sage Publications.

Bernal, Agusto (2006). Metodología de la investigación científica. 2. ${ }^{2}$ ed. Ed. Pearson Prentice Hall, p. 54-57

Burns, J.M. (1978). Leadership. USA: Harper and Row.

Cantera, J. NTP (2013). Satisfacción laboral: encuesta de evaluación. España: Ministerio de Trabajo y Asuntos Sociales, Instituto Nacional de Higiene y Seguridad en el Trabajo. Disponible en: http://www.insht.es. Consultado el 16 de abril de 2014.

Chiang, M., Salazar, M., Huerta, P., Nunez, A. (2008). Clima organizacional y satisfacción laboral en organizaciones del sector estatal (instituciones públicas). Desarrollo, adaptación y validación de instrumentos. Universum. Disponible en: http://www.scielo.cl. Consultado el 16 de abril de 2014.

Chiavenato, I. (2006). Introducción a la teoría general de la administración. 7. a ed. México D.F. McGraw-Hill Interamericana, p. 298.

Domínguez, Agustín. Los valores estructurales de la persona. México: Porrúa, 2001 $-2002.2 \mathrm{v}$.

Fernández, B., Paravic, T. (2003). Nivel de satisfacción laboral en enfermeras de hospitales públicos y privados de la provincia de Concepción, Chile. Ciencia y Enfermería; IX (2): 57-66.

Ferrater M., José. Diccionario de Filosofía. Madrid: Alianza, 1979 (reimp. 1986). 4 v. $3589 \mathrm{p}$.

Fiabane, E., Giorgi, I., Squazzin, C., Argentero, P. (2013). Work engagement and occupational stress in nurses and other healthcare workers: the role of organizational and personal factors. J. Clin Nurs. Doi:10.1111/jocn. 12084.

Galaz, J.F., Contreras, P. (2002). La satisfacción laboral de los académicos mexicanos en una universidad estatal pública: la realidad institucional bajo la lente del profesorado. Benito Juárez, México: ANUIES; 21-3. 
García, I. (2006). La formación de clima psicológico y su relación con los estilos de liderazgo. Granada: Universidad de Granada, Departamento de Psicología Social y Metodología de las Ciencias del Comportamiento. 112 p.

Gillespie, N. y Mann, L. (2000). The building blocks of trusts: the role of transformational leadership and shared values in predicting team members' trust in their leaders. Academy of Management, Conference. Disponible en http//www. mbs.unimelb.edu.au/jgans/wp/200001.pdf. Consultado el 3 de septiembre de 2014.

González, Lissette y col. (2013). Relación de la satisfacción laboral con estilos de liderazgo en enfermeros de hospitales públicos. Santiago, Chile. Revista Ciencia y Enfermería XIX (1): 11-21, ISSN 0717-2079.

Hernández, R., Fernández, C. y Baptista, P. (1994). Metodología de la investigación. Colombia: Mc Graw Hill.

Hersey, P., Blanchard, K., Johnson, D. (1998). Administración del comportamiento

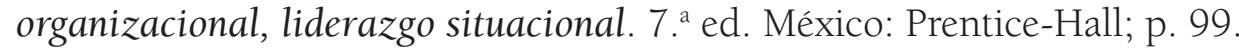

Judge, Piccolo e Ilies (2004). Personal Factors Associated with Leadership: A Survey of the Literature. Journal of Psychology. p 35-71.

Locke, E.A. (1987). The nature and causes of job satisfaction. En Dunnette MD, editor. Handbook of industrial and organizational psychology. Chicago: Rand-Mc Nally College; p. 297-349.

MacKenzie, S., Podsakoff, P. y Rich, G. (2001). Transformational and Transactional leadership and salesperson performance. Academy of Marketing Science Journal; volume 29, N. ${ }^{\circ}$ 2; p. 115-134.

Marín, I. Diego (2005). La enseñanza de las teorías de la administración: limitantes epistémicos y posibilidades pedagógicas. Disponible en http://www.bdigital. unal.edu.co/13042/1/38-208-1-PB.pdf. Fecha de acceso: 8 de julio de 2014.

Martínez, Eutimio (2009). Liderazgo. Análisis filosófico del liderazgo organizacional. Disponible en: http://www.gestiopolis.com/administracion-estrategia-2/ analisis-filosofico-del-liderazgo-organizacional.htm. Consultado el 8 de abril de 2014.

Martínez, Jonny (2009). Liderazgo. Disponible en: http://www.gestiopolis.com/administracion-estrategia/cuatro-fundamentos-del-liderazgo.htm. Fecha de acceso: 15 de septiembre de 2014.

Marriner-Tomey, A. (1998). Administración y liderazgo en enfermería. 5. a ed. Editorial: Harcourt Brace. p. 273-349.

Marriner-Tomey, A. (2009). Guía de gestión y dirección de enfermería. 8. a ed. Barcelona: Elsevier. p. 544.

Mendoza, Gonzalo y Jorge Ubeda (2011). El liderazgo y la actitud filosófica. Escuela de Filosofía de Madrid. Disponible en: http://www.revistaconsejeros. 
com/espaniol/opinion.asp?valor=61\&id=134\&anio=2011\&ultimoNumero=61\&lengua=1. Consultado el 23 de julio de 2014 .

Mintzberg, Henry y Col (1999). Liderazgo; Bilbao: Deusto, 250 p.

Muchinsky, P.M. (2009). Psicología aplicada al trabajo. 6. ${ }^{a}$ ed. España: Thomson. p. 271.

Munduate, Jaca (1999). La motivación en el trabajo. Servicio de Publicaciones del Ministerio de Trabajo y Seguridad Social, Madrid. España.

Newstrom, J. y Davis, K. (1991). Comportamiento humano en el trabajo: comportamiento organizacional. Editorial McGraw Hill, 8. ${ }^{a}$ edición.

Parra, S., Paravick, T. (2002). Satisfacción laboral en enfermeras (os) que trabajan en el sistema de atención médica de urgencia (SAMU). Ciencia y Enfermería; VIII (2): p. 37-48.

Peiró, J. M. y Meliá, J. L. (2000). La medida de la satisfacción laboral en contextos organizacionales: el cuestionario de satisfacción S20/23. Disponible en: http:// www.uv.es/meliajl/Research/Art_Satisf/ArtS20_23.PDF. Fecha de acceso: 2 de septiembre de 2014.

Pérez Bilbao, Jesús (2011). NTP 394: satisfacción laboral: escala general de satisfacción. Ministerio de Trabajo y Asuntos Sociales. España.

Robbin, S. M. (2006). Administration. Madrid, España: Prentice-Hall; 2006, p. 395-396.

Rodríguez-Gonzalo, A., Munoz-Lobo, M.J., Marzo-Martínez, A., Sánchez-Vicario, F. (2009). Liderazgo en grupos de trabajo enfermeros. Percepciones. Enferm. Clin. 19(4): 206-209.

Sánchez, C. Yomar (2008). Pensamiento administrativo I. Fundación Universitaria Luis Amigó. Facultad de Ciencias Administrativas, Económicas y Contables. Colombia. Disponible en: www.funlam.edu.com._Fecha de acceso: 1 de septiembre de 2014.

Siliceo, A., Alfonso (1999). Liderazgo, valores y cultura organizacional: hacia una organización competitiva. México: McGraw-Hill, c1999. 220 p.

Siliceo, A., Alfonso (2001). Liderazgo: ¿cómo quieres que te recuerden tus seguidores? México: McGraw-Hill, c. 64 p.

Stogdill, R.M., Bass, B. (1990). Handbook of leadership: a survey of the literature. 3. ${ }^{a}$ ed. New York: Simon-Schister.

Vargas, G. Germán (2011). La formación como eje. De las relaciones entre pedagogía y administración. Revista electrónica Forum Doctoral, número 3. Edición especial. Enero - abril de 2011. ISSN: 2027-2146. Disponible en: http://www.revistadocencia.cl/pdf/20100730181900.pdf. Consultado el 18 de junio de 2014. 
Vega, Carolina y Gloria Zavala (2004). Adaptación del cuestionario multifactorial de liderazgo (MLQ forma $5 X$ corta) de B. Bass y B. Avolio al contexto organizacional chileno. Memoria para optar al título de psicólogo. Facultad de Ciencias Sociales. Universidad de Chile. 